\title{
689.
}

\section{ON THE GEOMETRICAL REPRESENTATION OF IMAGINARY VARIABLES BY A REAL CORRESPONDENCE OF TWO PLANES.}

[From the Proceedings of the London Mathematical Society, vol. Ix. (1878), pp. 31-39. Read December 13, 1877.]

IN my recently published paper, "Geometrical Illustration of a Theorem relating to an Irrational Function of an Imaginary Variable," Proceedings of the London Mathematical Society, t. vIII. (1877), pp. 212-214, [627], I remark as follows:- "If we have $v$ a function of $u$ determined by an equation $f(u, v)=0$, then to any given imaginary value $x+i y$ of $u$ there belong two or more values, in general imaginary, of $v$; and for the complete understanding of th? relation between the two imaginary variables we require to know the series of values $x^{\prime}+i y^{\prime}$ which correspond to a given series of values $x+i y$ of $v, u$ respectively. We must, for this purpose, take $x, y$ as the coordinates of a point $P$ in a plane $\Pi$, and $x^{\prime}, y^{\prime}$ as the coordinates of a corresponding point $P^{\prime}$ in another plane $\Pi^{\prime \prime}$; - and $I$ then proceed to consider the particular case where the equation between $u, v$ is $u^{2}+v^{2}=a^{2}$, that is, where

$$
(x+i y)^{2}+\left(x^{\prime}+i y^{\prime}\right)^{2}=a^{2} .
$$

The general case is that of an equation $(*)(u, 1)^{m}(v, 1)^{n}=0$, where to each given value, real or imaginary, of $u$, there correspond $n$ real or imaginary values of $v$; and to each given value, real or imaginary, of $v$, there correspond $m$ real or imaginary values of $u$. And then, writing $u=x+i y$ and $v=x^{\prime}+i y^{\prime}$, and regarding $(x, y),\left(x^{\prime}, y^{\prime}\right)$ as the coordinates of the points $P, P^{\prime}$ in the two planes $\Pi, \Pi^{\prime}$ respectively, we have a real $(m, n)$ correspondence between the two planes; viz. to each real point $P$ in the first plane there correspond $n$ real points $P^{\prime}$ in the second plane, and to each real point $P^{\prime}$ in the second plane there correspond $m$ real points $P$ in the first plane. But such real correspondence of two planes does not of necessity arise from an equation between the two imaginary variables $u, v$; and the 
question of the real correspondence of two planes may be considered in itself, without any reference to such origin.

I was under the impression that the theory was a known one; but I have not found it anywhere set out in detail. It is to be noticed that, although intimately connected with, it is quite distinct from (and seems to me to go beyond) that of a Riemann's surface. Riemann represents the value $u,=x+i y$, by a point $P$ whose coordinates are $x, y$; but he considers $u^{\prime},=x^{\prime}+i y^{\prime}$, as a given imaginary value attached to the point $P$, without representing this value by a point $P^{\prime}$, coordinates $x^{\prime}, y^{\prime}$.

I proceed to consider the general theory of the real $(m, n)$ correspondence. Points in the first plane are denoted by the unaccented letters $P, Q, \ldots$; and the corresponding points in the second plane are in general denoted by the same letters accented; but there are, as will be explained, special points $V, W$ where the letters are interchanged; viz. to the points $V$ or $W$ in the first plane correspond points $W^{\prime}$ or $V^{\prime}$ in the second plane.

1. To a point $P$ there correspond in general $n$ distinct points $P^{\prime}$; and as $P$ varies continuously, each of the points $P^{\prime}$ also varies continuously.

2. There are certain points $V$ called branch-points (Verzweigungspunkte), such that to each point $V$ there correspond two united points, represented by $\left(W^{\prime}\right)$, and $n-2$ other distinct points $W^{\prime}$. The points $\left(W^{\prime}\right)$ are called cross-points, and the number of them is of course equal to that of the branch-points $V$.

It is throughout assumed that a point denoted by a letter other than $V$ is not a point $V$.

3. If the point $P$, moving continuously, describe a closed curve so as to return to its original position, then, if this curve includes within it no point $V$ (or all the points $V)^{*}$, each of the corresponding points $P^{\prime}$ will describe continuously a closed curve returning into its original position. Supposing that the curve described by $P$ is an oval (non-autotomic closed curve), and taking this to be in the first instance an indefinitely small oval, then the curves described by the points $P^{\prime}$ will in the first instance be each of them an indefinitely small oval; but it is worth while to notice how, as the oval described by $P$ increases, any one of the ovals described by a point $P^{\prime}$ may become autotomic; viz. if the oval described by $P$ passes through two points $Q, Q$ of the $m$ points $Q$ which correspond in the first plane to the same $Q^{\prime}$ in the second plane, then $Q^{\prime}$ will be a node in the closed curve described by that point $P^{\prime}$ which in the course of its motion comes to pass through $Q^{\prime}$. This curve is in general an inloop curve composed of two loops, one wholly within the other (united at the point $Q^{\prime}$ ), and such that they each include one and the same point $V^{\prime}$ (viz. $V^{\prime}$ is included within the inner loop): as to this, see post, Nos. 9 and 10. It will be observed that this node $Q^{\prime}$ is not a point $\left(W^{\prime}\right)$ nor any other special point of the second plane.

* The two cases of the closed curve including no point $V$, and including all the points $V$, are really identical, as the discontinuity at infinity may be disregarded. It is to be observed that, this being so, it follows that the number of the points $V$ must be even. 
4. Consider, as before, $P$ as describing a closed curve which does not include within it any point $V$, and the corresponding points $P^{\prime}$ as describing each of them a closed curve. As the curve described by $P$ approaches a point $V$, the curves described by two of the points $P^{\prime}$ will approach the corresponding point $\left(W^{\prime}\right)$; and when the curve described by $P$ passes through $V$, the curves described by the two points $P^{\prime}$ will unite together at this point $\left(W^{\prime}\right)$ as a node; viz. they will form a figure of eight*, the crossing being at the cross-point $\left(W^{\prime}\right)$, which corresponds to the branch-point $V$. And, corresponding to the closed curve described by $P$, we have this figure of eight (replacing two of the original $n$ closed curves), and $n-2$ closed curves described by the other points $P^{\prime}$.

5. Supposing, next, that the closed curve described by $P$ (instead of passing through the point $V$ ) includes within it the point $V$, then the figure of eight transforms itself into a twice-indented oval*. There are on this curve two of the points $P^{\prime}$ which correspond to the given point $P$; and as $P$, moving continuously in its closed curve, returns to its original position, the first of these points $P^{\prime}$, moving continuously along a portion of the curve, comes to coincide with the original position of the second point $P^{\prime}$; while the second point $P^{\prime}$, moving continuously along the remaining portion of the curve, comes to coincide with the original position of the first point $P^{\prime}$; viz. the two portions of the curve are described by the two points $P^{\prime}$ respectively. The curve may thus be regarded as a bifid curve, belonging to these two points $P^{\prime}$. And, corresponding to the closed curve described by $P$, we have this bifid curve belonging to the two points $P^{\prime}$, and $n-2$ single closed curves belonging to the other $n-2$ points $P^{\prime}$ respectively.

6. If the closed curve described by $P$ (including within it a point $V$ ) comes to pass through a second point $V$, the effect will be a new node at the corresponding point $\left(W^{\prime}\right)$; viz. at this point $\left(W^{\prime}\right)$ either the bifid curve unites itself with one of the single curves, or two of the single curves unite together, or the bifid curve there cuts itself. And, if the curve described by $P$ comes to include within it this second point $V$, then in the three cases respectively:- the bifid curve takes to itself the single curve, so that the system then is a trifid curve and $n-3$ single curves; or the two single curves give rise to a bifid curve, so that the system is two bifid curves and $n-4$ single curves; or, lastly, the bifid curve breaks up into two single curves, so that the system resumes its original form of $n$ single curves.

7. We thus see how the closed curve described by $P$, including within it certain of the points $V$, may be such as to have corresponding to it an $\alpha$-fid curve, a $\beta$-fid curve, \&c., $(\alpha+\beta+\ldots=n)$; viz. an $\alpha$-fid curve contains upon it $\alpha$ of the points $P^{\prime}$ which correspond to the original position of $P$; and then, as $P$ describes

* The name figure of eight refers to the case where the two curves which come to unite at $\left(W^{\prime}\right)$ are proper ovals (non-autotomic closed curves). They might have one or both of them a node or nodes, as explained in No. 3; and the term would then be inappropriate. And so, lower down, the name twiceindented oval is used to express the form into which a proper figure of eight is changed by the disappearance of the node. 
continuously its closed curve, returning to its original position, each of these points $P^{\prime}$ describes a portion of the $\alpha$-fid curve, passing from its original position to the original position of a point $P^{\prime}$ next to it upon the $\alpha$-fid curve; and the like as to a $\beta$-fid curve, \&c. The numbers $\alpha, \beta, \ldots$ are not of necessity unequal, and we may have sets of equal numbers in any manner. It is hardly necessary to remark that, if the curve described by $P$ passes through any point or points $V$, then two of the curves described by the points $P^{\prime}$ will unite together, or it may be that one of these will cut itself at the corresponding point or points $\left(W^{\prime}\right)$; and further that, as in No. 3, if the curve described by $P$ passes through two or more of the points $Q$ which correspond to the same point $Q^{\prime}$, then any such point $Q^{\prime}$ will present itself as a node upon the curve belonging to some point, or set of points, $P^{\prime}$. But the order of succession in which the original $n$ single curves unite themselves together into multifid curves, or again break up into single curves, cannot, it would appear, be explained in any general manner, and would in each case depend on the nature of the particular correspondence.

8. We may consider the case where the closed curve described by $P$ cuts itself. The curve may here be considered as made up of two or more ovals, or, to use a more appropriate term, say loops, each such loop being a curve not cutting itself; and the case is thus reducible to that before considered, where the curve does not cut itself. Thus, to fix the ideas, let the curve be a figure of eight, the initial position of $P$ being at the crossing, and let neither of the loops contain within it a point $V$. Then, as $P$ passes continuously along one of the loops, returning to its original position, each of the corresponding points $P^{\prime}$ describes a closed curve, which will be in the nature of a loop, viz. the initial and final directions of the motion of $P$ not being continuous with each other, the initial and final directions of the motions of each point $P^{\prime}$ will not be continuous with each other, or there will be at the point $P^{\prime}$ an abrupt change in the direction of the curve. Similarly, as $P$ describes the other loop of the figure of eight, each of the points $P^{\prime}$ will describe another loop; and the two loops belonging to the same point $P^{\prime}$ will unite together so as to form a figure of eight; viz. to the figure of eight described by $P$ there will correspond figures of eight described by the $n$ points $P^{\prime}$ respectively.

9. But consider next the case where the two loops of the curve described by $P$ include each of them one and the same point $V$. This implies that one of the two loops lies inside the other, or that the curve is what has been called an inloop curve. As $P$, which is in the first instance taken to be at the node, passes continuously along one of the loops and returns to its original position, there are two of the points $P^{\prime}$ such that the first of these passes from its original position to the original position of the second, and the second of them passes from its original position to the original position of the first of them. We have thus two arcs between these two points $P^{\prime}$; but inasmuch as the initial and the final directions of motion of the point $P$ are not continuous with each other, these two arcs are not continuous in direction at the two points $P^{\prime}$, but at each of these points $P^{\prime}$ the two arcs meet at an angle, As $P$ describes the other loop, we have in like manner two arcs between the same two points $P^{\prime}$, these arcs at each of the points $P^{\prime}$ meeting at an 
angle; but they join on to the first-mentioned two arcs in such manner as to form two ovals intersecting each other in the two points $P^{\prime}$. Corresponding to the inloop curve described by $P$, we have this pair of intersecting ovals described by two of the points $P^{\prime}$, and $n-2$ other curves described by the other points $P^{\prime}$, and being each of them (I assume) an inloop curve.

10. If we attend only to one of the two intersecting ovals, we have in the first plane an inloop curve, and corresponding thereto in the second plane an oval passing through two of the points $P^{\prime}$ which correspond to the node $P$ of the inloop curve. Interchanging the two planes, and writing $Q$ instead of $P$, we have in the first plane an oval passing through two of the points $Q$ which correspond to a point $Q^{\prime}$; and corresponding to this oval we have in the second plane an inloop curve having this point $Q$ for its node, viz. these are the corresponding figures mentioned in No. 3.

11. Consider a given point $Q$; and let the corresponding points $Q^{\prime}$ be called (selecting the suffixes at pleasure) $Q_{1}^{\prime}, Q_{2}^{\prime}, \ldots, Q_{n}{ }^{\prime}$. Taking then a point $O$ indefinitely near to $Q$, the corresponding points $O^{\prime}$ will be indefinitely near to $Q_{1}^{\prime}, Q_{2}^{\prime}, \ldots, Q_{n}^{\prime}$ respectively, and they will be called $O_{1}^{\prime}, O_{2}{ }^{\prime}, \ldots, O_{n}{ }^{\prime}$ accordingly. It is to be observed that by the indefinitely near point $O$ is meant a point such that the distance from $O$ to $Q$ is indefinitely small in comparison with the distance of either of these points from any point $V$; so that we cannot have from $Q$ to $O$ two indefinitely short paths including between them a point $V$; or say so that the indefinitely short path from $Q$ to $O$ is determinate.

Proceeding in this manner from $Q$ to $O$, and so through a succession of indefinitely near points to a distant point $S$, we seem to determine the suffixes of the corresponding points $S^{\prime}$; but, by what precedes, it appears that such determination for a point $S$ is dependent on the path from $Q$ to $S$; and consequently that we do not thus obtain a proper determination of the suffixes of the points $S^{\prime}$. In fact, if we were to pass from $Q$ by a path including one or more of the points $V$ back to $Q$, we should obtain for the several points $Q^{\prime}$ respectively suffixes which are in general different from the suffixes originally given to these points respectively.

12. The difficulty is got over as follows:-Considering as before the given point $Q$, and calling the corresponding points $Q_{1}{ }^{\prime}, Q_{2}{ }^{\prime}, \ldots, Q_{n}{ }^{\prime}$ at pleasure, we pass from $Q$ to the indefinitely near point $O$, and thence, by so many paths chosen at pleasure, to the several branch-points $V$; these paths from $O$ to the several points $V$ are called barriers. To fix the ideas, we may consider these as non-autotomic non-intersecting lines drawn from $O$ to the several points $V$. Consider the barrier from $O$ to one of these points $V$; as $P$ passes along this barrier from $O$ to $V$, two of the corresponding points $P^{\prime}$ will pass from two of the corresponding points $O^{\prime}$ to the corresponding cross-point $\left(W^{\prime}\right)$; the paths of these two points are called the counter-barrier corresponding to the barrier in question; and we have thus in the second plane a system of counter-barriers, each drawn from two points $O^{\prime}$ to meet in a point $\left(W^{\prime}\right)$. By what precedes, the points $O^{\prime}$ have each of them a determinate suffix; a counterbarrier is thus drawn from two points with given suffixes, suppose $O_{1}^{\prime}$ and $O_{2}^{\prime}$, to a 
point $\left(W^{\prime}\right)$, and this may be distinguished accordingly as a counter-barrier 12; and in like manner the cross-point $\left(W^{\prime}\right)$ through which it passes will be called a crosspoint $\left(W_{12}{ }^{\prime}\right)$; and the barrier corresponding hereto, and the branch-point $V$ at which it terminates, will in like manner be called a barrier 12, and a branch-point $V_{12}$. Each barrier and branch-point will thus have a pair of suffixes; and the corresponding counter-barrier and cross-point will have the same pair of suffixes. It is to be observed that two or more of these corresponding figures may very well have the same pair of suffixes; but that such corresponding figures must be distinguished from each other; thus, if there are two branch-points $V_{12}$, these may be distinguished as the branch-points $\alpha V_{12}$ and $\beta V_{12}$, and the barriers, counter-barriers, and cross-points by means of these same letters $\alpha$ and $\beta$, (or otherwise), as may be convenient. It would seem that not only the number of the points $V$ must be even, but the number of each set of points $V_{12}$ must also be even (see post, No. 15).

13. It is also to be noticed that the determination of the suffixes of the several points $V$, \&c., depends first upon the arbitrary choice of the suffixes of the points $Q^{\prime}$, and next on the choice of the system of barriers; but that, these being assumed, the suffixes of the several points $V$, \&c., are completely determinate.

14. Taking now any point $S$ whatever, and supposing that $P$ moves from $Q$ continuously to $S$ by a path which does not meet a barrier, the points $P^{\prime}$ will move from the several points $Q^{\prime}$ to the several points $S^{\prime}$ by paths not meeting the counterbarriers; viz. to each point $S^{\prime}$ there will be a path from some point $Q^{\prime}$; and giving to such point $S^{\prime}$ the suffix of the point $Q^{\prime}$, the suffixes of the several points $S^{\prime}$ which correspond to any point whatever, $S$, will be completely determined. The determination depends of course on the assumptions referred to No. 13, but not in anywise on the position of the point $S$.

It will be noticed that, as all the points $V$ are connected together by the barriers, the only closed paths from a point to itself are paths not including any, or including all, of the points $V$; and that between such paths there is no real distinction.

15. Consider a point $P$ moving continuously in any manner. The several corresponding points $P_{1}^{\prime}, P_{2}^{\prime}, \ldots, P_{n}^{\prime}$ will each of them move continuously, but the suffixes interchange; viz. when $P$ arrives at and then passes over a barrier $\alpha \beta$, the corresponding points $P_{a}^{\prime}$ and $P_{\beta}{ }^{\prime}$ will each arrive at the corresponding counter-barrier $\alpha \beta$, and, on passing over this, $P_{\alpha}^{\prime}$ will be changed into $P_{\beta}^{\prime}$ and $P_{\beta}^{\prime}$ into $P_{\alpha}^{\prime}$, the other points $P^{\prime}$ remaining unchanged; and the like in other cases. This in fact includes the whole or the greater part of the foregoing theory. Thus, if $P$ describe a closed curve not cutting any barrier, there will be no change of suffix; and when $P$ returns to its original position each of the corresponding points $P_{1}^{\prime}, P_{2}^{\prime}, \ldots, P_{n}^{\prime}$ will describe a closed curve, returning to its original position. But suppose that $P$ describes a closed curve, cutting once only a barrier 12 ; suppose that the path is from $P$ to $Q$, and then crossing the barrier to $R$, and thence again to $P ; P_{1}^{\prime}$ passes to $Q_{1}^{\prime}$, and then crossing the counter-barrier it passes from $R_{2}^{\prime}$ to $P_{2}^{\prime}$; while at the same time $P_{2}^{\prime}$ passes to $Q_{2}^{\prime}$, and then crossing the counter-barrier it passes from $R_{1}^{\prime}$ to $P_{1}^{\prime}$;

c. $\mathbf{x}$. 
viz. we have $P_{1}^{\prime}, P_{2}^{\prime}$ describing the two portions of a bifid curve. If there were only a single branch-point $V_{12}$, and therefore only a single barrier $O V_{12}$, then we might have through $P$ a closed curve cutting $O V_{12}$ once only, and including within it the point $O$, but not including within it the point $V_{12}$; and here there ought not to be a bifid curve, but the points $P_{1}^{\prime}, P_{2}^{\prime}$ ought to describe each of them a single curve. But suppose there are two points $V_{12}$, and consequently two barriers $O V_{12}$ (meeting in 0 ); then the closed curve, meeting once only a barrier 12, (viz. it meets only one such barrier, and that once only), must include within one and only one of the two points $V_{12}$; and in this case there ought to be a bifid curve. It is by such reasoning as this that I infer the foregoing theorem (No. 12), that the number of each set of points $V_{12}$ is even.

16. We may consider how the suffixes are affected when, instead of the original system of barriers, we have a new system of barriers. I suppose that we have in the two cases respectively the same point $Q$, and the same suffixes for the points $Q_{1}^{\prime}, Q_{2}^{\prime}, \ldots, Q_{n}^{\prime}$ which correspond thereto. In the first case, passing from $Q$ to an indefinitely near point $O$, say the red $O$, we draw from this point to the several points $V$ a set of barriers, say the red barriers; while in the second case, passing from $Q$ to an indefinitely near point $O$, say the blue $O$, we draw from this point to the several points $V$ a set of barriers, say the blue barriers; and we then proceed as before, viz. in the first case, drawing from $Q$ to the point $S$ a curve which does not meet any of the red barriers, we determine accordingly the suffixes (say the red suffixes) of the several corresponding points $S^{\prime}$; and in the second case, drawing in like manner from $Q$ to $S$ a curve which does not meet any of the blue barriers, we determine accordingly the suffixes (say the blue suffixes) of the same points $S^{\prime}$. Now the curve drawn from $Q$ to $S$ so as not to cut any of the red barriers, and which is used for the determination of the red suffixes of the several points $S^{\prime}$, will in general cut certain of the blue barriers; and, by examining the suffixes of the blue barriers which are thus cut, we determine the blue suffixes of the same points $S^{\prime}$; the result of course depending only on the situation of $S$ in one or other of the regions formed by the red barriers and the blue barriers conjointly. In particular, the point $S$ may be so situate that we can from $Q$ to $S$ draw a curve not meeting any red barrier or any blue barrier; and in this case the red suffixes and the blue suffixes are identical.

17. We may imagine the first plane as consisting of $n$ superimposed planes or sheets, say the sheets $1,2, \ldots, n$. Each barrier 12 is considered as a line drawn in the two sheets 1 and 2 ; and so on in other cases. The point $P$ is considered as a set of superimposed points $P_{1}, P_{2}, \ldots, P_{n}$ moving in the several sheets respectively; under the convention that $P_{1}$ moving in the sheet 1 , and coming to cross a barrier 12, passes into the sheet 2 and becomes $P_{2}$; and the like in other cases. And this being so, we say that to a point $P$, considered as a point $P_{a}$ in the sheet $\alpha$, there corresponds in the second plane one and only one point $P_{a}{ }^{\prime}$; and that $P$ moving continuously in any manner (subject to the change of sheet as just explained), each of the $n$ corresponding points $P^{\prime}$ will also move continuously, and so that each such point $P_{a}^{\prime}$ will return 
to its original position, upon the corresponding point $P_{a}$ returning to its original position and sheet. This is, in fact, Riemann's theory, only instead of the points $P^{\prime}$ we must speak of the values $x^{\prime}+i y^{\prime}$ of the irrational function of $x+i y$.

18. Everything is of course symmetrical as regards the two planes; we have therefore, in the second plane, a system of points $V^{\prime}$ and of barriers, and in the first plane a system of points $(W)$ and of counter-barriers. To a given point $P^{\prime}$ in the second plane there correspond $m$ points $P$ in the first plane; and we can (the determination depending on the system of barriers in the second plane) assign to the $m$ points suffixes, thereby distinguishing them as the corresponding points $P_{1}, P_{2}, \ldots, P_{m}$. And we may imagine the second plane as consisting of $m$ superimposed planes or sheets, say the sheets $1,2,3, \ldots, m$; the general theorem then is that to a point $P$ or $P^{\prime}$ in either plane, considered as a point $P_{a}$ or $P_{a}^{\prime}$ in the sheet $\alpha$ or $\alpha^{\prime}$, there corresponds in the other plane one and only one point $P_{a}^{\prime}$ or $P_{a^{\prime}}$; and that the firstmentioned point in either plane moving continuously in any manner (subject to the proper change of sheet), the corresponding point in the other plane will also move continuously, and will return to its original position and sheet, upon the firstmentioned point returning to its original position and sheet.

19. In all that precedes it has been assumed that, to a branch-point $V$, there correspond two united points represented by $\left(W^{\prime}\right)$ and $n-2$ distinct points $W^{\prime}$; the cases of a point $\left(W^{\prime}\right)$ composed of three or more united points, or of the points $W^{\prime}$ uniting themselves in sets in any other manner, would give rise to further specialities. 